\title{
Archiv der Pharmazie
}

301. Band; Heft 1, Seite 33-38

Verlag Chemie, GmbH, Weinheim//Bergstr.

W. Wiegrebe, D. Sasse und E. Roesel

Überführung von 1-Benzylisochinolinen in Berbinium-Salze

5. Mitt.: Ein Vergleich des Coralyn-Ions mit dem 2,3,9,10-Tetramethoxy-5-methyldibenzo[b, $\mathbf{g}]$-chinolizinium-Ion 
, 
W. Wiegrebe, D. Sasse und E. Roesel

\section{Überführung von 1-Benzylisochinolinen in Berbinium-Salze}

\section{Mitt.: Ein Vergleich des Coralyn-Ions mit dem 2,3,9,10-Tetramethoxy-5-methyl- dibenzo $[b, g]$-chinolizinium-Ion*)}

Aus dem Institut für Pharmazeutische Technologie der Techn. Hochschule Braunschweig

(Eingegangen am 14. Juli 1967)

Es wird eine Synthese C-5-substituierter Dibenzo[b, g]-chinolizinium-Salze beschrieben. Ein Vergleich des Coralyn-Ions mit dem 2,3,9,10-Tetramethoxy-5-methyl-dibenzo[b,g]-chinclizinium-Ion zeigt, daß diese Ionen nicht miteinander identisch sind.
We report a synthetic route to C-5-substituted dibenzo[b, g]-quinolizinium-salts. A comparison of coralyn-ion and 2,3,9,10tetramethoxy -5 - methyl-dibenzo[b, g]-quinolizinium-ion shows that these ions are not identical.

Bei der Umsetzung von Papaverin (I) mit Acetyl-Kationen entsteht ein ImoniumSalz ${ }^{1}$ ), das zum Berbin-Derivat Coralydin (II), reduziert werden kann. Daraus folgerten Schneider und Schroeter ${ }^{1}$ ), daß dieses Imonium-Salz, das „Coralyn “, ebenfalls eine Berbin-Struktur (III, 2,3,9,10-Tetramethoxy-8-methyl-dibenzo[a,g]-chinolizinium-Verbindung) hat.<smiles>COc1cc2c(cc1OC)C(C)N1CCc3cc(OC)c(OC)cc3C1c1cc(OC)c(OC)cc1C2</smiles>

Die Coralyn-Formel im Sinne einer Dibenzo[a,g]-Verbindung wurde zweifelhaft, als es nicht gelang, ein am C-3 methyliertes 1-Benzylisochinolin, das sog. „Alteupaverin“(IV), zu einem Coralyn-Derivat umzusetzen $\left.{ }^{2}\right)$. W. $A w e^{3}$ ) stellte daraufhin die Arbeitshypothese auf, Coralyn sei kein Berbin-, sondern ein Paraberin-Derivat (V). Diese These wurde in der vorstehenden Mitt.*) besprochen und widerlegt. Es blieb jedoch notwendig, das Coralyn III mit der für diese Verbindung vorgeschlagenen Struktur V direkt zu vergleichen, um die Verschiedenartigkeit beider Substanzen deutlich zu machen.

*) 4. Mitt. vorstehend.

1) W. Schneider und K. Schroeter, Ber. dtsch. chem. Ges. 53, 1459 (1920).

2) W. Awe, Pharmaz. Zentralhalle Deutschland 77, 157 (1936).

3) Angew. Chem. 59, 32 (1947). 
<smiles>Cc1cc2cc3c(cc2c(Cc2ccc4c(c2)OCO4)n1)OCO3</smiles><smiles>COc1ccc(CC2Cc3cc(OC)c(OC)cc3C=N2)cc1OC</smiles><smiles>CI</smiles><smiles>COc1ccc(CC2Cc3cc(OC)c(OC)cc3CN2)cc1OC</smiles>

IX<smiles>COc1ccc(Cc2cc3cc(OC)c(OC)cc3cn2)cc1OC</smiles>

VI

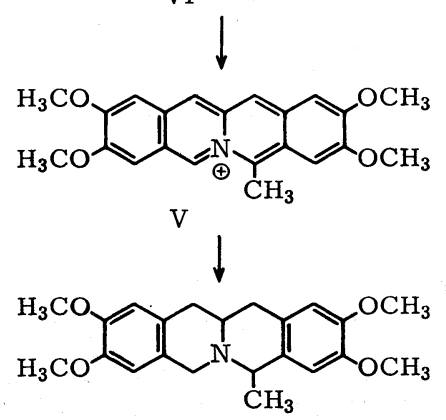

VIII

Bradsher $^{4}$ ) hat erstmalig Dibenzo[b,g]-chinolizinium-Salze durch Cyclodehydrierung von 3-Formyl-N-veratryl-isochinolinium-Salzen hergestellt. Dieser Weg ist u. E. für die Synthese von V wegen der Substitution am C-5 ungeeignet: eine Quaternisierung des 6,7-Dimethoxy-isochinolin-3-aldehyds mit 1-Brom-1-(3',4'-dimethoxyphenyl)-äthan hätte voraussichtlich sehr schlechte Ausbeuten geliefert, denn sie wäre nicht nur durch die Substitution des C-3 des Isochinolins, sondern auch durch die Verzweigung des quaternisierenden Agens erschwert (vgl. ${ }^{5}{ }^{6}$ )). Hinzu kommt bei der Paraberin-Synthese nach Bradsher die Gefahr einer Dimerisierung ${ }^{4}$ ).

Wir haben daher das dem Coralyn III isomere Paraberin V durch Acetylierung des 6,7-Dimethoxy-3-veratryl-isochinolins (VI) gewonnen. Voraussichtlich ist dieser Weg zur Synthese C-5-substituierter Paraberine allgemein gangbar. VI wurde durch Dehydrierung des entsprechenden 3,4-Dihydroisochinolins VII her-

$\left.{ }^{4}\right)$ C. K. Bradsher und T. W. G. Solomons, J. Amer. chem. Soc. 82, 1808 (1960).

$\left.{ }^{5}\right)$ H. C. Brown, D. Gintis und H. Podall, J. Amer. chem. Soc. 78, 5376 (1956).

$\left.{ }^{6}\right) H$. Decker und $O$. Klauser [Ber. dtsch. chem. Ges. 37, 3812 (1904)] fanden, daß die Umsetzung von I mit Isopropyljodid sehr niedrige Ausbeuten liefert. 
gestellt ${ }^{7}{ }^{8}$ ). Bei der Umsetzung von VI mit Sulfoessigsäure bildete sich schnell eine leuchtend rot gefärbte, kristalline Verbindung. Durch Reduktion mit Zink und Säure entstand daraus eine farblose Hexahydro-Base VIII, die identisch mit der Verbindung war, die wir durch saure Kondensation aus 6,7-Dimethoxy-3-veratryl1,2,3,4-tetrahydroisochinolin $\mathrm{IX}^{7}$ ) mit Acetaldehyddiäthylacetal gewonnen hatten. Diese Übereinstimmung stützte die Annahme, daß das erwähnte rote Kristallisat $\mathrm{V}$ ist. Bewiesen wurde diese Annahme durch das 100-MHz-NMR-Spektrum dieses Salzes (Abb. 1, TMS als innerer Standard, $\mathrm{F}_{3} \mathrm{C}-\mathrm{COO} \mathrm{D}$ als Lösungsmittel): im Bereich der aromatischen Protonen treten nur Singulette auf!
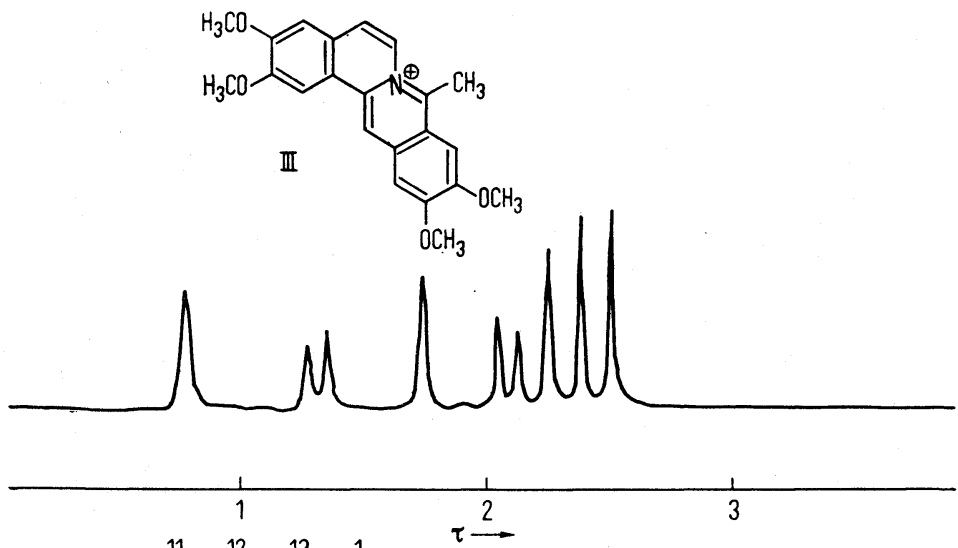

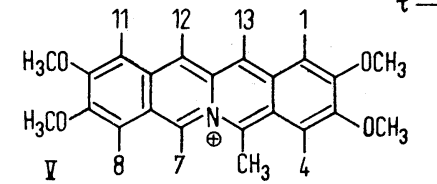

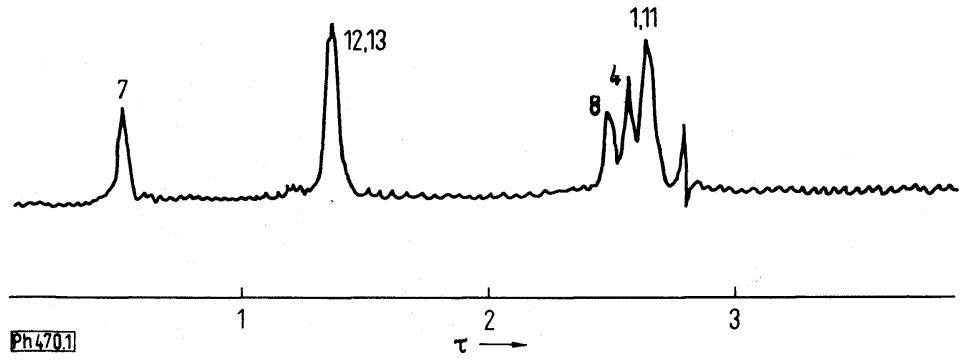

Abb. 1

7) S. Sugasawa, K. Kakemi und H. Kazumi, Ber. dtsch. chem. Ges. 73, 782 (1940).

$\left.{ }^{8}\right)$ J. Knabe und N. Ruppenthal, Arch. Pharmaz. 297, 268 (1964). 
Mit wachsender Feldstärke erscheinen die Signale folgender Protonen: $\mathrm{H}_{7}-\mathrm{H}_{12}$, $\mathrm{H}_{13}-\mathrm{H}_{8}-\mathrm{H}_{4}-\mathrm{H}_{1}, \mathrm{H}_{11}$. Demgegenüber enthält das Spektrum des Coralyns III, das zusammen mit den NMR-Spektren analoger Verbindungen in einer weiteren Veröffentlichung dieser Reihe eingehend besprochen wird, das AB-System, wie es für o-ständige Protonen zu fordern ist.

Die UV-Spektren des Coralyns III und des isomeren Paraberins V zeigt Abb. 2. Erwartungsgemäß absorbiert V langwelliger als III.

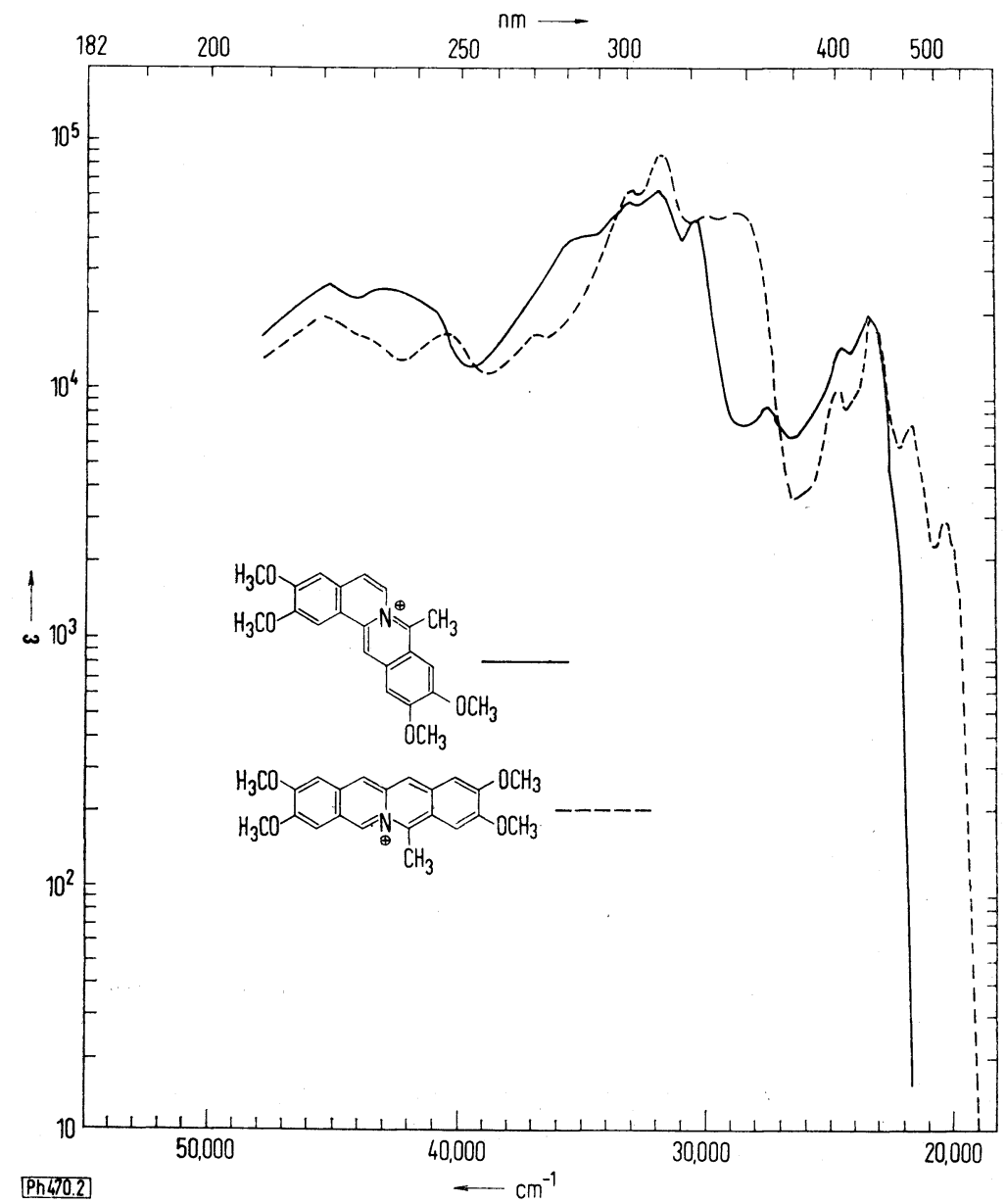

Abb. 2

Das Fluoreszenzlicht des Coralyns (III) hat in Methanol oder Methanol/HCl ein Maximum bei $479 \mathrm{~nm}$. In 0,5 $\mathrm{n} \mathrm{HCl}$ (wäßrige Lösung) emittiert III ein Fluoreszenzlicht mit einem Maximum bei $516 \mathrm{~nm}$. 
Eine neutrale methanolische Lösung von V strahlt ein Fluoreszenzlicht mit 2 Maxima aus (510 und $542 \mathrm{~nm}$ ). In neutraler oder saurer wäßriger Lösung erscheint nur ein Maximum bei $507 \mathrm{~nm}$ (die Messungen in wäßrigen Lösungen sind ungenau; da V sich nur sehr schwer in Wasser löst).

Dem Land Niedersachsen sind wir für die Förderung dieser Arbeit durch Forschungsmittel zu Dank verpflichtet. :

\section{Beschreihung der Versuche}

6,7-Dimethoxy-3-veratryl-3,4-dihydroisochinolin (VII) wurde nach $\left.{ }^{7}\right)^{8}$ ) hergestellt. Versuche, die einzelnen Syntheseschritte zu verbessern, werden in den Diss. $E$. Roesel und D. Sasse beschrieben.

\section{6,7-Dimethoxy-3-veratryl-isochinolin (VI)}

$4,7 \mathrm{~g}$ VII wurden in $25 \mathrm{ml}$ frisch destilliertem Tetralin gelöst, mit 0,5 g Pd-Mohr (aus Contactol der Fa. Doduco) versetzt und 5 Std. im Metallbad bei $220^{\circ}$ (Badtemp.) erhitzt. Nach dem Abkühlen versetzten wir das Reaktionsgemisch mit so viel Äther, daß eine einheitliche org. Phase entstand und filtrierten das Pd ab. Aus dem Filtrat wurde die Base durch Ausschütteln mit $\mathrm{n} \mathrm{HCl}$ extrahiert, die salzsaure Lösung erhitzt und durch Filtrieren geklärt. Durch Zugabe von 10proz. KOH fiel die Base aus, die in Äther aufgenommen wurde. Durch Einleiten von HCl-Gas in die getrocknete Ätherlösung fiel ein schwach gelb gefärbter, z. T. kristalliner Niederschlag aus, der mehrmals aus Aceton/Methanol $95+5$ am Durchlaufextraktor umkristallisiert wurde. Schmp. des VI-Hydrochlorids: 186-187 .

$$
\begin{array}{lll}
\mathrm{C}_{20} \mathrm{H}_{22} \mathrm{NO}_{4} \mathrm{Cl}(375,9) & \text { Ber.: C 63,91 } & \text { H 5,90 } \\
& \text { Gef.: C 63,50 } & \text { H 5,92 }
\end{array}
$$

Aus dem VI-Hydrochlorid konnte die Base durch Zugabe von 10proz. Ammoniak freigesetzt werden. Sie kristallisierte aus Äther in feinen Nadeln. Die DC in verschiedenen Fließmitteln auf Kieselgel G nach Stahl der Fa. E. Merck (Aceton 65, Methanol 20, Wasser 15 oder Benzol - Methanol 9 : 1) zeigte, daß die Base rein war. Schmp. 110-111 ${ }^{\circ}$, Ausbeute: durchschnittlich $51 \% \mathrm{~d}$. Th.

$$
\begin{array}{lll}
\mathrm{C}_{20} \mathrm{H}_{21} \mathrm{NO}_{4}(339,4) & \text { Ber.: C 70,78 } & \text { H 6,23 } \\
& \text { Gef.: C 70,69 } & \text { H 6,08 }
\end{array}
$$

\section{Umsetzung von VI zum Dibenzo[b,g]-chinolizinium-Sulfoacetat V}

$0,8 \mathrm{ml}$ konz. Schwefelsäure und 4,0 ml Acetanhydrid wurden auf dem Wasserbad so lange erhitzt, bis in der braunroten Mischung keine Sulfat-Ionen mehr nachweisbar waren ${ }^{\mathbf{1}}$ ). Wir gaben 1,0 g VI hinzu, und nach wenigen Min. hatte sich ein roter Kristallbrei gebildet. Wir erhitzten noch 10 Min., ließen abkühlen und verrührten die Mischung langsam mit Methanol, um überschüssiges Acetanhydrid umzusetzen. Daraufhin wurden die Kristalle getrocknet. Das Rohprodukt, dessen NMR-Spektrum gemessen wurde, konnte aus viel Methanol umkristallisiert werden. Das reine Produkt fiel in verfilzten, feinen Nädelchen aus. Die methanol. Lösung fluoreszierte gelbgrün.

$$
\begin{array}{lll}
\mathrm{C}_{24} \mathrm{H}_{25} \mathrm{NO}_{9} \mathrm{~S}+{ }^{1} /{ }_{2} \mathrm{CH}_{3} \mathrm{OH}(519,5) & \text { Ber.: C 56,64 } & \text { H 5,24 } \\
& \text { Gef.: C 56,81 } & \text { H 5,56 }
\end{array}
$$

m IR-Spektrum des umkristallisierten Produktes war Methanol nachweisbar: bei $3400 \mathrm{~cm}^{-1}$ trat eine breite Bande auf. 


\section{Redaktion von V zu VIII}

$0,5 \mathrm{~g} \mathrm{~V}$ wurden in der Mischung aus $30 \mathrm{ml}$ 5proz. Schwefelsäure, $15 \mathrm{ml}$ Eisessig und 2,5 ml wasserfreier Ameisensäure mit Zinkpulver unter Rückfluß erbitzt. - Nach 1 Std. wurde vom Zink abfiltriert, das Zink noch zweimal mit 15proz. Essigsäure ausyekocht, die Base nach dem Abkühlen mit Ammoniak gefällt und mit Äther ausgeschüttelt. Nach dem Einengen kristallisierte die Base aus. Das DC (Benzol 8, Aceton 1, Methanol 1) zeigte, daß noch geringfügige Verunreinigungen vorhanden waren. Die Base wurde dalier wieder in Äther gelöst, mit 0,5 $\mathrm{n} \mathrm{HCl}$ ausgeschüttelt, die salzsaure Phase nit Talcum geklärt und die Base, die jetzt de rein war, kristallisiert. Ausbeute: $70 \%$ d. Th. Schmp. $198^{\circ}$ (Zers.).

$$
\begin{array}{lll}
\mathrm{C}_{22} \mathrm{H}_{27} \mathrm{NO}_{4}(369,4) & \text { Ber.: C 71,52 } & \text { H 7,37 } \\
& \text { Gef.: C 71,33 } & \text { H 7,40 }
\end{array}
$$

\section{Unabhängige Synthese von VIII}

Die Hydrierung von VII mit katalytisch erregtem Wasserstoff ${ }^{7}$ ) (Pt aus Actiplat der Fa. Doduco als Katalysator) lieferte IX $\left.{ }^{7}\right)$. 0,5 g IX-Hydrochlorid wurden in $5 \mathrm{ml} 15$ proz. HCl gelöst und auf dem Wasserbad im Laufe von 4 Std. mit $3 \mathrm{ml}$ Acetaldehyddiäthylacetal versetzt. - Nach dem Erkalten wurde die saure Lösung mit Äther ausgeschüttelt, die Base mit Ammoniak gefällt und in Äther aufgenommen. Nach dem Filtrieren über $\mathrm{Al}_{2} \mathrm{O}_{3}$, standardisiert nach Brockmann, kristallisierte die Base beim Einengen aus. Sie wurde wie oben beschricben - gereinigt und schmolz dann bei 197-198 ${ }^{\circ}$ (Zers.). Ausbeute: $64 \%$ d. Th. Das IR-Spektrum dieser Base war identisch mit dem der Base, die aus V entstanden war. Beide Substanzen gaben im Mischschmp. keine Depression.

\section{Allgemeine Angaben}

Die Schmp. wurden im Kupferblock oder auf dem Kofler-Heiztisch bestimmt und sind unkorrigiert angegeben.

Die IR-Spektren wurden im Beckman-Gerät IR-5, die UV-Spektren mit dem ZeissSpektralphotometer PMQ II aufgenommen. Die Fluoreszenz-Spektren wurden mit dem Fluoreszenzansatz ZFM 4 zum PMQ II, Aufstellung A, bestimmt. Die NMR-Sjektren wurden mit dem Gerät HA 100 der Fa. Varian gemessen.

Herrn Prof. Dr. Awe danken wir vielmals für seine Unterstützung. Unser verbindlicher Dank gilt Herrn Prof. Dr. Zinner und Herrn Dr. Ritter für die IR-Spektren. Herrn Prof. Dr. Dr. h. c. Inhoffen, Herrn Dr. Krüger und Frl. Koch schulden wir Dank für die NMRSpektren. Die Firmen E. Merck AG. und Doduco-Chemie haben diese Arbeit dankenswerterweise mit Chemikalien- bzw. Katalysator-Spenden unterstützt. 